\title{
Multifocal Skeletal Tuberculosis Involving the Lumbar Spine and Iliac Bone, Mimicking a Malignant Bone Tumour: A Case Report
}

\author{
Gosal GS, MS Orth, Boparai A, MD Path, Choudhary G, MD Radio, Kour R, MD Anaest \\ Department of Orthopaedics, Chintpurni Medical College, Pathankot Punjab, India
}

\begin{abstract}
Multifocal osteoarticular tuberculosis is uncommon and accounts for approximately $10 \%$ of all cases of osteoarticular tuberculosis. Concomitant vertebral and iliac bone tuberculosis has not been reported in the literature to date. We report one such case for its unusual clinical and radiological presentation, which had presentation more similar to a malignant bone tumour than an infection.
\end{abstract}

Key Words:

Skeletal tuberculosis, multifocal osteoarticular tuberculosis

\section{INTRODUCTION}

Osteoarticular tuberculosis is uncommon and accounts for $2 \%$ of all cases of systemic tuberculosis ${ }^{1}$. While $50 \%$ of osteoarticular tuberculosis occurs in the vertebral column, less frequently affected sites are the hip, knee and sacroiliac joints ${ }^{1}$. The multifocal form of skeletal tuberculosis is exceptional, with a rate of $10 \%$ of all skeletal tuberculosis cases even in countries where the disease is endemic ${ }^{1,5}$. Multifocal skeletal lesions may occur as a result of hematogenous dissemination from another primary focus such as cervical lymph nodes, lungs, tonsils or gastrointestinal tract. Iliac bone tuberculosis is rare and presentation with concomitant vertebral tuberculosis is even more rare, and has not been reported to date in the literature. We report the case of a patient with lumbar vertebral and iliac bone tuberculosis presenting as a malignant bone tumour rather than infection.

\section{CASE REPORT}

A 25-year-old immunocompetent man presented with lower back pain and discomfort in the left gluteal region for the previous 3 months; the pain was insidious in onset and progressive. The pain was aggravated by sitting, walking and lying on the ipsilateral side. There were no associated constitutional symptoms and no fever. There was no obvious swelling or sinus, and no evidence of any systemic illness. At the L3 vertebral level and left iliac wing, there was tenderness. No reflex, sensory, or motor changes were noted in the lower limbs.
Blood tests showed an increased erythrocyte sedimentation rate of $50 \mathrm{~mm}$ in the first hour and normal total cell count as well as differential cell count. A radiograph of the lumbar spine revealed sclerosis of the L3 vertebral body with destruction and excavation of the anterior half of the vertebral body but with sparing the peridiscal region (Figure 1). Radiographs of the pelvis showed that the entire left ilium had a large multicystic lytic expansile lesion with a few areas of sclerosis but sparing of the sacroiliac joint (Figure 2). A bone scan did not reveal any other lesions. Radiography and ultrasonography of the abdomen were unremarkable and ELISA for Human Immunodeficiency Virus was negative. Magnetic resonance imaging (MRI) of the pelvis showed a large expansile lesion involving the left iliac and ischial bone with broken cortices at multiple sites which showed as heterogeneously hyperintense signal in T2 weighted images (Figure 3A,B) and STIR sequence (Figure 3C) with a large hyperintense soft tissue component involving gluteus muscle, iliacus muscle, rectus abdominis muscle and lower part of left erector spinae muscle. A peripheral enhancement pattern was seen on post contrast images (Figure 3D). Extensive cortical destruction was seen, although the sacroiliac joint and sacrum were not involved. MRI of the spine showed altered signal intensity at the L3 vertebral body with a T2 hyperintense demarcated lesion in the body with a small anterior soft tissue component and destruction of anterior vertebral cortex. End plates of the L3 vertebra were intact.

An open incisional biopsy of the left iliac bone was performed which revealed caseous material. A closed vertebral biopsy of L3 vertebral lesion was also performed. Polymerase chain reaction (PCR) assays for mycobacterium tuberculosis were positive from both lesions. Histopathological examination revealed chronic granulomatous inflammation with central caseous necrosis, infiltration of epithelioid cells and Langerhans giant cells, all suggestive of tuberculosis. The patient was prescribed antitubercular chemotherapy with isoniazid, rifampicin, pyrazinamide, and ethambutol for three months and was advised to be on bed rest. Three months afterwards, he was asymptomatic and his ESR dropped to $19 \mathrm{~mm} / \mathrm{hr}$. Antitubercular chemotherapy was continued with isoniazid and rifampicin for 12 more months. At the final follow-up, the

Corresponding Author: Gurinder Singh Gosal, Department of Orthopaedics, Chintpurni Medical College and Hospital, Pathankot, Punjab, India Email: gurindergosal@yahoo.co.in 


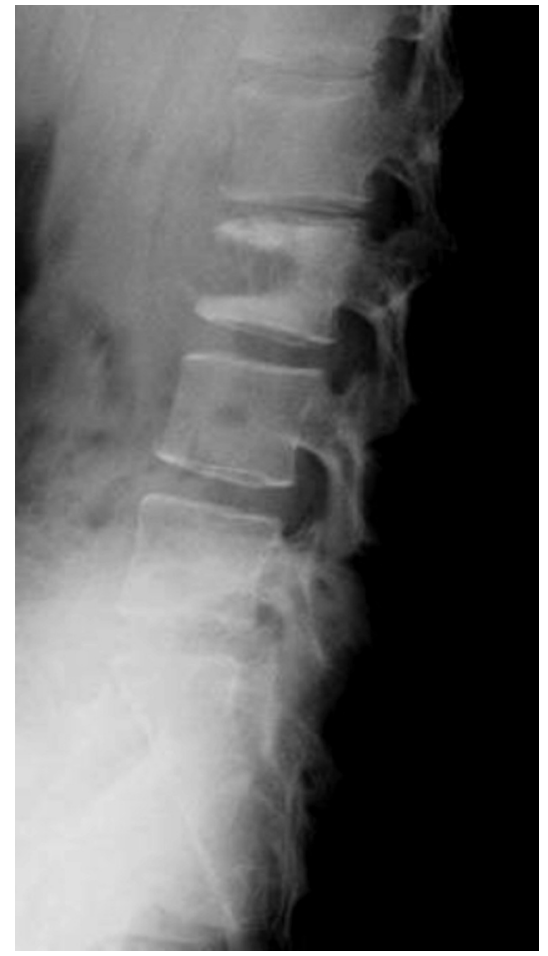

Fig. 1: A radiograph of the lumbar spine showing sclerosis of the L3 vertebral body with destruction and excavation of the anterior half of the vertebral body but with sparing the peridiscal region.

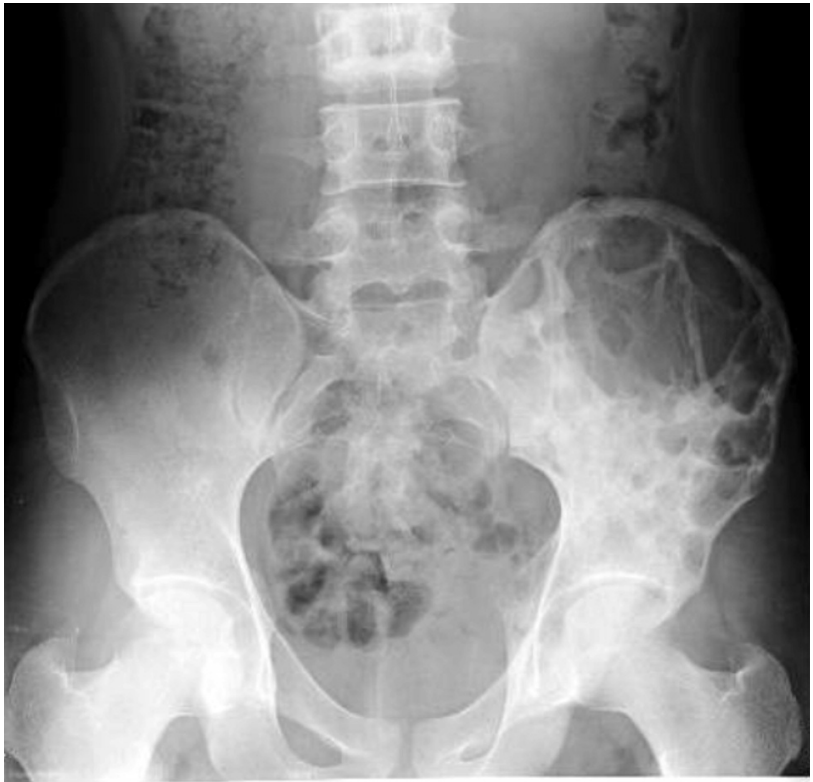

Fig. 2: Radiograph of the pelvis showing the entire left ilium with a large multicystic lytic expansile lesion with a few areas of sclerosis but sparing of the sacroiliac joint.
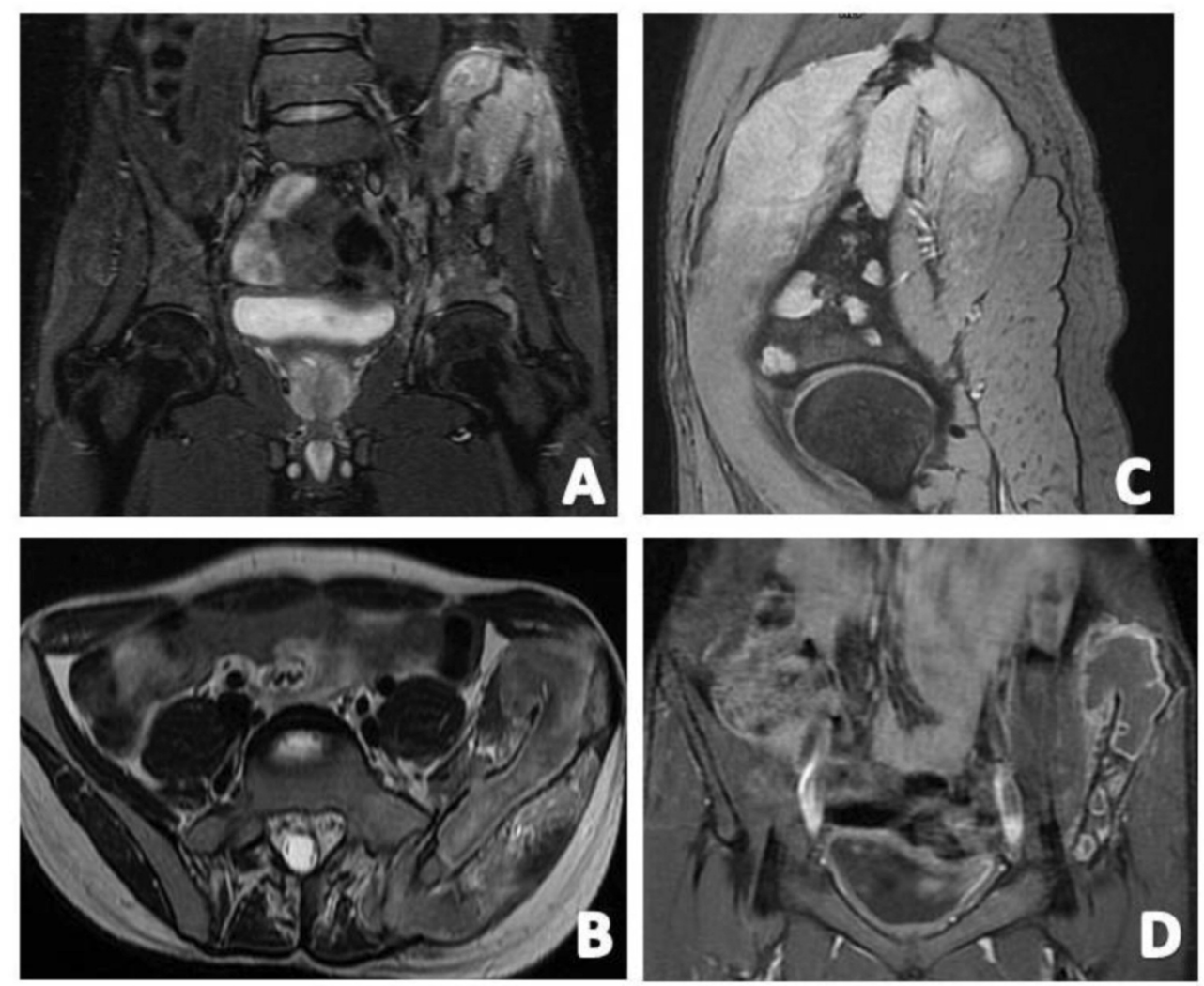

Fig. 3: Magnetic resonance imaging (MRI) of the pelvis showed a large expansile lesion involving the left iliac and ischial bone with broken cortices at multiple sites which showed as heterogeneously hyperintense signal in T2 weighted images (Panels A, B) and STIR sequence (Panel C) with a large hyperintense soft tissue component involving gluteus muscle, iliacus muscle, rectus abdominis muscle and lower part of left erector spinae muscle. A peripheral enhancement pattern was seen on post contrast images (Panel 3D). 
patient was free of symptoms, though there was little change in the radiological picture except increased sclerosis of the lesion suggestive of radiological healing.

\section{DISCUSSION}

Tuberculosis is still highly prevalent in developing countries like India. The organs primarily involved in tuberculosis are cervical lymph nodes, lungs, tonsils and gastrointestinal tract, but all other organs in the body may get secondarily involved from any of these primary sites. Osteoarticular tuberculosis accounts for $2 \%$ of patients with tuberculosis with the most common site being the spine $(50 \%)$, and the second most common being the hip joint $(20 \%)$ followed by the knee joint $(10 \%)$. Tuberculosis of bones and joints is characterized by monoarticular involvement, progressive symptoms, muscle wasting and constitutional symptoms. Although multifocal involvement is rare $(10 \%)$, there have been sporadic case reports of such lesions especially in immunocompromised patients. Most cases of multifocal tuberculosis reported in the literature have been either continuous or skipped spinal lesions ${ }^{1,5}$. Multifocal tuberculosis, brought about by disseminated haematogenous impregnation, is found almost exclusively in those with a compromised immune status ${ }^{14}$. However, concomitant multifocal tuberculosis with spinal and iliac bone involvement has not been reported to date. Moreover, the radiological picture of the lesions was remarkable. The earliest radiological sign of vertebral tuberculosis is typically localized osteoporosis and the most common radiological presentation is peridiscal metaphyseal erosion. In this case however, there was a vertebral osteolytic lesion with excavation and some loculi involving the anterior half of the body of the L3 vertebra, destruction of the anterior cortex of the vertebral body and sclerosis of the remaining portions of the L3 vertebra. Multiple osteolytic lesions with a honeycomb appearance of the entire left ilium sparing the sacroiliac joint further confused the clinical and radiological picture. Considering the radiographic and MRI features we at first believed that a tumour was the likely diagnosis and went ahead with biopsy of the lesion. However, histopathology and PCR confirmed multifocal skeletal tuberculosis as the final diagnosis. Multifocal skeletal tuberculosis should be considered as a differential diagnosis in cases presenting with multiple bone lesions. Antituberculosis chemotherapy usually leads to healing, provided the diagnosis is early, which is possible only if a high index of suspicion is maintained.

\section{REFERENCES}

1. Tuli SM. Tuberculous osteomyelitis: Tuli SM, editor. Tuberculosis of the Skeletal System. 2nd ed.,chapter 1, New Delhi: Jaypee Brothers, 2000.

2. Goldblatt M, Cremin BJ. Osteo-articular tuberculosis: its presentation in coloured races. Clin Radiol. 1978; 29 : 669-77.

3. Dhammi IK, Jain AK, Singh S, Aggarwal A, Kumar S. Multifocal skeletal tuberculosis in children: a retrospective study of 18 cases. Scand J Infect Dis. 2003; 35: 797-9.

4. Morris BS, Varma R, Garg A, Awasthi M, Maheshwari M. Multifocal musculoskeletal tuberculosis in children: appearances on computed tomography. Skeletal Radiol. 2002; 31: 1-8.

5. Moore SL, Rafii M. Imaging of musculoskeletal and spinal tuberculosis. Radiol Clin North Am. 2001; 39: 329-42. 